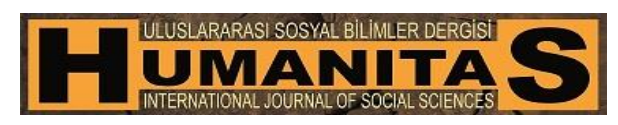

Humanitas, 2017; 5(9): 29-43

ISSN: 2147-088X http://humanitas.nku.edu.tr

DOI: $10.20304 /$ humanitas.286529

Araştırma-İnceleme

\title{
TRADUCTION EN TURC DE L'ARTICLE 5 DE LA CONVENTION EUROPÉENNE DES DROITS DE L'HOMME: DIFFÉRENTS TYPES DE LA DÉTENTION
}

\begin{abstract}
Deniz KURMEL ${ }^{1}$
Résumé: La traduction a une place importante pour le domaine des droits de l'homme européen puisque les langues officielles de la Convention européenne des droits de l'homme et de la jurisprudence de la Cour européenne des droits de l'homme sont l'anglais et le français. Les états membres de la Convention transfèrent ces textes dans leurs langues via la traduction. Dans la traduction des droits de l'homme européen, la transposition des concepts ayant des caractéristiques relatifs à ce domaine mérite une réflexion; les nouvelles approches terminologiques qui prennent en compte les termes dans leur environnement discursif peuvent offrir une méthodologie dans la traduction des droits de l'homme, surtout dans le transfert des termes du domaine qui prennent leur sens dans les textes de la jurisprudence. Dans cet article, nous problématisons la traduction des droits de l'homme européen en turc; à travers la traduction des différents types du terme de détention qui est le terme clé de l'article 5 de la Convention européenne des droits de l'homme «Droit à la liberté et à la sûreté ». Nous adoptons une méthodologie terminologique textuelle basée sur l'analyse contextuelle menée dans des corpus composés des textes de jurisprudence en français et en turc, afin d'examiner des différents types de détention tels que; détention provisoire, détention préventive, détention sans jugement, détention administrative et leurs traductions en turc.
\end{abstract}

Mots-clés: Traduction des droits de l'homme européen, terminologie textuelle, analyse contextuelle, analyse du corpus, détention.

\footnotetext{
1 Arş. Gör. Dr., Yııldız Teknik Üniversitesi, Fen Edebiyat Fakültesi, Fransızca Mütercim Tercümanlık Bölümü. dkocak@ yildiz.edu.tr
} 
Kurmel, D. (2017). Traduction en Turc de l'article 5 de la convention Europeenne

des droits de l'homme:differents yypes de la detention. Humanitas, 5(9), 29-43

\section{AVRUPA INSSAN HAKLARI SÖZLEŞMESI'NIN 5. MADDESININ ÇEVIRISII: TUTUKLULUK (DÉTENTION) TERIMININ FARKLI TÜRLERI}

Öz: Avrupa İnsan Hakları Sözleşmesi ve Avrupa İnsan Hakları Mahkemesi içtihatlarının resmi dili İngilizce ve Fransızca olduğundan, Avrupa İnsan Hakları alanında çevirinin önemli bir yeri vardır. Sözleşmeye taraf olan ülkeler, ilgili metinleri dillerine çeviri yoluyla aktarmaktadır. Avrupa İnsan Hakları alanının çevirisinde, alana özgü bazı özelliklere sahip olan kavramların aktarımı üzerinde durulması gereken bir konudur. Bu noktada, terimleri bulundukları söylem boyutunun içerisinde ele alan yeni terimbilim yaklaşımları söz konusu alanın çevirisinde, özellikle de içtihat metinlerinde anlam kazanan terimlerin aktarımında, yöntemsel bir çerçeve sunabilir. Bu makalede, Avrupa İnsan Haklarının Türkçe'ye çevirisini, ve ilgili sözleşmenin 5. Maddesi olan "Özgürlük ve Güvenlik Hakkı"nın anahtar terimi tutuklamanın (détention) türleri üzerinden tartısmaktayız. Oluşturduğumuz Fransızca ve Türkçe içtihat metinleri bütüncelerinde yürüttü̆̆ümüz bağlamsal çözümlemeye dayalı metinsel terimbilim yöntemiyle détention provisoire, détention préventive, détention sans jugement, détention administrative gibi farklı tutukluluk (détention) türlerini ve bu türlerin Türkçeye çevirilerini ele almaktayız.

Anahtar Sözcükler: Avrupa İnsan Haklarının Çevirisi, Metinsel Terimbilim, Bağlamsal Çözümleme, Bütünce Çözümlemesi, Tutuklama.

\section{Introduction}

Les droits de l'homme constituent un sujet important et discuté depuis des siècles. Les philosophes, les politiciens ont réfléchi sur ce sujet à toutes les époques. Mais c'est surtout après le désastre que l'humanité a vécu pendant la deuxième Guerre Mondiale, que l'idée de créer un système basé sur le respect des droits de l'homme a vu le jour.

Après la deuxième Guerre Mondiale, l'idée de fonder une nouvelle Europe est née afin de défendre et de promouvoir la liberté et la démocratie, et c'est dans ce but qu'en 1949, le Conseil de l'Europe a été fondé. Il a tout de suite commencé à travailler sur la Convention de sauvegarde des droits de l'homme et cette Convention plus connue sous le nom de «Convention européenne des droits de l'homme ", a été ouverte à la signature à Rome le 4 novembre 1950 et est entrée en vigueur le 3 septembre 1953. La Convention a concrétisé certains des droits et libertés inscrits dans la Déclaration universelle des droits de l'homme et créé une juridiction internationale compétente pour condamner les États ne respectant pas leurs engagements. Les Etats ayant ratifié la Convention, qu'on appelle aussi "Etats parties à la Convention », reconnaissent et garantissent les droits fondamentaux civils et politiques non seulement à leurs ressortissants, mais également à toute personne relevant de leur juridiction. 
Sudre (2006) souligne que l'originalité de la Convention européenne réside moins dans la liste des droits énumérés que dans le mécanisme institutionnel de protection mis en place, qui a son siège à Strasbourg. La Convention est un document qui évolue surtout grâce à l'interprétation de ses dispositions par la Cour européenne des droits de l'homme. Avec sa jurisprudence, la Cour fait de la Convention un instrument vivant; elle a ainsi élargi les droits garantis et a permis leur application à des situations qui n'étaient pas prévisibles lors de l'adoption de la Convention.

La Convention européenne des droits de l'homme a été signée le 4 novembre 1950 par la Turquie, qui l'a ratifiée en adoptant une « Loi sur la ratification de la Convention de sauvegarde des droits de l'homme et libertés fondamentales et du protocole annexe» (numéro 6366) le 10 mars 1954. Le document de ratification de la Convention a été déposé au Secréterait Général du Conseil de l'Europe le 18 mai 1954 et à partir de cette date elle est entrée en vigueur pour la Turquie (Gemalmaz, 2003). Le protocole 11 à la Convention qui restructure le mécanisme de contrôle judiciaire a été signé le 11 mai 1994 et ratifié le 11 juillet 1997 par la Turquie. Ce protocole est en vigueur pour la Turquie depuis le $1^{\text {er }}$ novembre 1998. Depuis que la Turquie est devenue un état membre de la Convention, elle a été condamnée un nombre considérable de fois par la Cour européenne des droits de l'Homme.

\section{Contexte en question}

Les documents de base des droits de l'homme européen se sont transposés en turc par le biais de traductions puisque les langues officielles de la Convention européenne et de la Cour européenne des droits de l'homme sont l'anglais et le français. Donc, on peut dire que la traduction est un acte central dans la conception de la langue des droits de l'homme en turc. En tant qu'un chercheur dans le domaine de traduction, dans cet article, nous voulons problématiser la traduction des droits de l'homme européen en turc; à travers la traduction des différents types du terme de détention qui est le terme clé de l'article 5 de la Convention européenne des droits de l'homme «Droit à la liberté et à la sûreté».

L'article 5 de la Convention représente un composant essentiel du système de protection des droits individuels et vise à protéger la liberté physique de la personne contre toute arrestation et détention arbitraire et abusive (Engel et al. c. Pays-Bas, 8 juin 1976, GACEDH, ${ }^{\circ} 4$, § 58) (Sudre, 2006). L'article 5 est entouré de deux concepts clés de la privation de liberté; détention et arrestation. La détention que notre recherche se focalise, dénommée aussi comme la détention provisoire ou détention préventive ou encore détention avant jugement ${ }^{2}$, est une privation de liberté qui commence avec la décision d'un organe judiciaire. Dans le Dictionnaire des droits de l'homme, Damien Roets définit ainsi détention provisoire ; "la mesure par laquelle un magistrat

\footnotetext{
${ }^{2}$ Equivalents mentionnés dans le Dictionnaire des droits de l'homme (Andriantsimbazovina et $a l ., 2008)$ au sein de l'entrée de la détention provisoire en tant que synonymes.
} 
Kurmel, D. (2017). Traduction en Turc de l'article 5 de la convention Europeenne

des droits de l'homme:differents yypes de la detention. Humanitas, 5(9), 29-43

prive de sa liberté une personne soupçonnée d'avoir commis une infraction pénale avant toute décision juridictionnelle la déclarant coupable des faits qui lui sont reprochés» (2012, p.271).

L'exécution de l'article 5 «Droit à la liberté et à la sûreté » constitue un des sujets les plus discutés en Turquie, il est possible de parler d'une relationnalité entre le transfert en turc de cet article, et plus spécialement celui de la détention, et son exécution.

\section{Approche méthodologique}

Le sujet de la traduction et de la terminologie des droits de l'homme a déjà été traité par de nombreux chercheurs comme Garre (1999), Chodkiewicz et al. (2002), Baccouche (2001; 2004), Alouini (2003), Pic, (2007a) (2007b) (2008), Cortese (2005), Peshkov (2012), Kurmel (2014).

En général, les travaux basés sur la terminologie et la traduction des droits de l'homme soulignent le caractère flou des termes de la Convention européenne des droits de l'homme qui ont d'une part permis son acceptation par plusieurs pays et, d'autre part, laissent à la jurisprudence de la Cour européenne des droits de l'homme une certaine liberté d'interprétation (Peshkov, 2012). Dans la traduction des droits de l'homme, le problème essentiel relève quasi exclusivement des questions terminologiques, puisque les difficultés se concentrent sur la traduction des concepts du domaine des droits de l'homme comme Pic le souligne (2007b). Donc, les nouvelles approches terminologiques qui prennent en compte les termes dans leur environnement discursif peuvent offrir une méthodologie dans la traduction des droits de l'homme, surtout dans le transfert des termes du domaine qui prennent leur sens dans les textes de la jurisprudence.

La terminologie textuelle proposée par Bourrigault et Slodzian (1999) décrit le terme dans le texte ; "le texte est le point de départ de la description lexicale à construire. On va du texte vers le terme ». Le terme est un construit ; «Il est le produit d'un travail d'analyse, mené par le linguiste terminologue, dont les choix sont guidés par une double contrainte de pertinence. Pertinence vis-à-vis $d u$ corpus [...] Pertinence vis-à-vis de l'application [...]» (Bourigault et Slodzian, 1999, p.31). La terminologie textuelle se rapproche de la linguistique, et notamment de la linguistique de corpus. Ils mettent l'accent sur le changement pratique et théorique de la terminologie ;

«[...] l'activité de construction d'une terminologie est désormais essentiellement une tâche d'analyse de corpus textuels. Ils appellent du même coup à un renouvellement théorique de la terminologie: c'est dans le cadre d'une linguistique textuelle que doivent être posées les théoriques de la terminologie. » (1999, p.30)

Avec cette optique, notre recherche se fonde sur l'analyse contextuelle réalisée dans le corpus des textes des droits de l'homme européen en français et en turc. 
Kurmel, D. (2017). Traduction en Turc de l'article 5 de la convention Europeenne des droits de l'homme:differents yypes de la detention. Humanitas, 5(9), 29-43

\section{Transposition en turc des textes des droits de l'homme européen}

Puisque les textes du domaine se transposent en turc par le biais des traductions, dans cette section nous traitons les travaux de traduction en turc des droits de l'homme européen. Une fois qu'un État ratifie la Convention et ses protocoles, il doit les traduire dans sa langue et les publier dans le Journal Officiel. Ces traductions officielles sont des textes qui sont en vigueur dans le système judiciaire national. La traduction officielle de la Convention en turc est publiée dans le Journal Officiel du 19 mars 1954, numéro 8662. Après la ratification du Protocole 11 à la Convention, la traduction de ce protocole est publiée dans le Journal Officiel du 20 juin 1997, numéro 23025. A part le changement des articles concernant le mécanisme du contrôle judiciaire, ce protocole indique les intitulés d'articles à insérer dans le texte. Nous constatons aussi l'existence des traductions plus récents, mais non officielles faites plutôt par les spécialistes des droits de l'homme (e.g. Osman Doğru, Mehmet Semih Gemalmaz). Ces traductions n'ayant pas de valeur juridique dans le système national mais ayant un but de transférer la Convention en turc d'une façon plus cohérente, méritent une réflexion plus détaillée et peuvent être un sujet d'un autre article.

La Convention prend son sens au sein de la jurisprudence de la Cour européenne des droits de l'homme comme nous avons déjà traitée, c'est-à-dire les concepts de la Convention sont interprétés dans des situations concrètes par la Cour. Les langues officielles de la jurisprudence de la Cour sont aussi l'anglais et le français. Il n'est pas obligatoire de faire une traduction officielle de la jurisprudence dans les langues des Etats contractants, mais ces traductions sont nécessaires pour que les autorités judiciaires nationales prennent en compte les décisions de la Cour pour éviter toute violation de la Convention. Dans l'application effective de la Convention européenne des droits de l'homme, la traduction de la jurisprudence de la Cour est une étape essentielle afin que chacun puisse comprendre les concepts et le fonctionnement de la Convention.

En Turquie, le Ministère de la Justice réalise des travaux de traduction de la jurisprudence ; le premier but du Ministère est de faire connaitre les décisions concernant la Turquie afin d'empêcher que les mêmes types de violations se répètent. Il transmet les décisions de la Cour dans le périodique publié depuis l'année 2004 par la Direction du Cercle d'études du Ministère de la Justice ${ }^{3}$ intitulé Yargı Mevzuatı Bülteni (Bulletin de législation judiciaire). Ces traductions sont plutôt des résumés en turc des décisions de la Cour. Le Ministère de la Justice a fondé, le 8 août 2011, le Département des droits de l'homme ${ }^{4}$ sous la Direction générale du droit international et de la relation étrangère ${ }^{5}$. Ce département qui travaille pour éliminer les causes de violations de la Convention dirige aussi les travaux de traduction de la jurisprudence, qui

\footnotetext{
${ }^{3}$ TC. Adalet Bakanlığı Eğitim Daire Başkanlığı

${ }^{4}$ İnsan Hakları Daire Başkanlığ 1

${ }^{5}$ Uluslararası Hukuk ve Dış İlişkiler Genel Müdürlüğü
} 
ont un caractère plus systématique puisqu'ils ne se limitent pas avec les arrêts contre la Turquie.

Les traductions réalisées au sein du Ministère de la Justice commencent à être diffusées dans la base de données HUDOC $^{6}$, portail de recherche de la Cour européenne des droits de l'homme, qui permet d'accéder à la jurisprudence de la Cour européenne des droits de l'homme (arrêts de Grande Chambre, arrêts de chambre et de comité, décisions, affaires communiquées, avis consultatifs et résumés juridiques de la Note d'information sur la jurisprudence), de la Commission européenne des droits de l'homme (Décisions et rapports) et du Comité des Ministres (résolutions).

Il est évident de rappeler l'existence d'une initiative non-officielle ; «Projet des droits de l'Homme - la jurisprudence de la Cour européenne des droits de l'Homme», dirigée par le juriste spécialisé dans le domaine des droits de l'homme, Osman Doğru. Ce projet nous donne l'occasion d'accéder à un nombre important de traductions en turc de la jurisprudence ${ }^{7}$.

\section{Description du corpus}

Dans cet article, nous analysons la traduction des différents types du concept clé de l'article 5 qui est la détention dans deux corpus comparables; corpus de jurisprudence de la Cour européenne en français et en turc limités avec les arrêts concernant l'article 5. Premièrement, nous créons le corpus en français à l'aide de la base de données HUDOC, portail de recherche de la Cour européenne des droits de l'homme. Nous avons accédé à 293 décisions d'arrêts en français ${ }^{8}$ concernant l'article 5, qui date de 1961 à 2010 et nous les avons placés dans notre corpus de jurisprudence en français La taille de ce corpus est de 3.323.068 mots. Deuxièmement, pour que nous puissions mener à bien une analyse contextuelle, nous constituons un corpus de jurisprudence en turc qui contient les mêmes textes que le corpus de jurisprudence en français. Dans la construction d'un tel corpus en turc, nous constatons qu'une base de données exhaustive n'existe pas en turc dans le domaine des droits de l'Homme, pour cette raison nous collectons le corpus en turc à partir des traductions du Ministère de la Justice et du Projet des Droits de l'Homme dirigé par le juriste Osman Doğru. Dans la catégorie des traductions ministérielles, nous n'avons que 32 traductions d'arrêts contre la Turquie concernant l'article 5 entre les années 1995-2008 ${ }^{9}$. Et dans la catégorie des traductions faites au sein du projet nous accédons à 42 arrêts concernant l'article 5 traduits en turc entre les années 19612005. Au total, nous avons 74 arrêts en turc, et la taille de ce corpus est de 392. 281 mots. Nous menons notre recherche dans le cadre de ces 74 arrêts qui existent en turc et en français. Il convient de souligner que dans le cadre des traductions en

\footnotetext{
${ }^{6}$ http://hudoc.echr.coe.int

${ }^{7}$ http://ihami.anadolu.edu.tr

${ }^{8}$ Ce corpus est collecté dans le cadre de la thèse de doctorat (non publiée) (Kurmel, 2014).

${ }^{9} \mathrm{La}$ collecte du corpus est réalisée avant le 8 août 2011 qui est la date de la fondation du Département des droits de l'homme ${ }^{9}$ au sein du Ministère de la Justice.
} 
turc, la langue du texte de départ n'est pas indiquée et les deux textes authentiques, français et anglais, font également foi et peuvent influencer la traduction.

La démarche outillée que nous utilisons s'inscrit dans la lignée de celle définie dans le cadre de la terminologie textuelle. De fait, les termes sont considérés comme les pivots de notre analyse et constituent le point d'entrée sur lequel s'appuie l'analyse. La description est menée à l'aide d'outils classiques en terminologie textuelle, c'est le concordancier AntConc. AntConc ${ }^{10}$ est un logiciel libre développé par L. Anthony (2005). Nous avons choisi ce concordancier pour plusieurs raisons: premièrement, il s'agit d'un logiciel gratuit, facile d'installation et d'utilisation. Il permet de visualiser les termes en contextes, mais également de construire des expressions régulières très fines pour affiner les recherches, de calculer les cooccurrences, etc. Et la langue turque est définie dans ce logiciel au sein des codes de langues de la norme ISO (8859-9) et cette qualité d'AntConc permet de pouvoir travailler sans problème avec le corpus en turc.

\section{Analyse contextuelle de la détention dans les corpus de la jurisprudence en français et en turc}

A partir d'une analyse contextuelle concernant le terme détention dans notre corpus de jurisprudence en français, nous remarquons l'existence de certains syntagmes qui nous amènent à d'autres types de détention comme; détention provisoire (219 occurrences), détention préventive (210 occurrences), détention sans jugement (9 occurrences), détention administrative (2 occurrences).

Une étude contextuelle, de ces termes complexes que nous postulons comme des différents types du terme détention, met en lumière l'emploi et le cadre sémantique de ces termes en question. Nous constatons que certains hyponymes ont peu d'occurrences dans le corpus comme détention sans jugement, détention administrative, mais nous analysons aussi ces termes dans leur contexte afin de ne pas négliger un terme significatif en éliminant les hapax ou les quasi -hapax.

A partir de notre analyse dans le corpus français, nous menons une recherche dans le corpus turc sur la traduction de ces types de détention. Cette étude sur la traduction des termes complexes en turc nous montre que les traducteurs adoptent des stratégies de communication dans le choix des équivalents, nous constatons l'existence d'une stratégie d'implicite et d'explicite.

\subsection{Détention provisoire}

Détention provisoire est un terme complexe qui, comme son nom l'indique, souligne le caractère provisoire de la détention qui se déroule dans l'attente d'un jugement. Ce terme a un nombre d'occurrences très significatif dans le corpus de jurisprudence en français. Quand nous faisons une requête dans le corpus de jurisprudence en turc avec l'équivalent turc de l'adjectif provisoire qui est geçici

\footnotetext{
${ }^{10}$ Le concordancier est disponible à l'adresse : http://www.antlab.sci.waseda.ac.jp/software.html (consulté en janvier 2010)
} 
Kurmel, D. (2017). Traduction en Turc de l'article 5 de la convention Europeenne des droits de l'homme:differents yypes de la detention. Humanitas, 5(9), 29-43

nous obtenons les syntagmes geçici tutma (9 occurrences) et geçici olarak tutulma ( 2 occurrences) avec des nombres d'occurrences minimes par rapport au corpus français.

A partir de cette différence importante au niveau du nombre d'occurrences entre le corpus français et le turc, nous pouvons dire que le terme complexe: détention provisoire n'est pas toujours rendue en turc de la même façon qu'en français, c'est-à-dire avec la combinaison de l'équivalent de détention et de provisoire. En général, ce terme complexe est traduit en turc avec les équivalents en turc de détention sans employer le modifieur temporel. On peut prétendre que l'implicitation, qui est un "résultat d'une 'économie' qu'on obtient en ne reformulant pas explicitement dans le 'texte d'arrivée' des éléments d'information $d u$ 'texte de départ' quand ils ressortent de façon évidente du 'contexte' ou de la 'situation' décrite et sont présupposés par les locuteurs de la 'langue d'arrivée' » (Delisle et al., 1999, p. 44), est généralement adoptée comme une stratégie de communication dans la traduction du terme complexe détention provisoire puisque le caractère provisoire est rendu en turc implicitement.

L'affaire De Jong, Baljet et Van Den Brink contre Pays-Bas (1984) tiré de nos corpus de jurisprudence (Tableau 1) nous montre que le terme tutukluluk (détention), qui est une des variantes terminologiques en turc de détention, peut être utilisé comme équivalent de la détention provisoire. L'emploi des variations terminologiques en turc de détention sans employer l'adjectif provisoire est en question, c'est-à-dire sans préciser le caractère provisoire de façon explicite, mais d'ailleurs il faut souligner que le terme détention et ses équivalents en turc comme tutma, tutuklama, gözaltında tutma, tutukluluk ont un caractère provisoire qui sont implicites puisqu'ils font référence à une privation de liberté à titre temporaire. Le lecteur turc devrait deviner le caractère provisoire de la détention partant du contexte ou de la situation de cette affaire.

Tableau 1: Affaire De Jong, Baljet et Van Den Brink contre Pays-Bas (1984) et sa traduction en turc

\begin{tabular}{|l|l|}
\hline «e procès eut lieu le 6février 1980 & «Askeri Mahkemedeki yargılama 6 \\
devant le conseil de guerre qui, le & Şubat 1980 tarihinde başlamıştır. Askeri \\
20, déclara M. van den & Mahkeme 20 Şubat tarihinde verdiği \\
Brink coupable et lui infligea dix- & kararla, Van den Brink'i suçlu bulmuş ve \\
huit mois d'emprisonnement, avec & on sekiz ay hapis cezast vermiş, \\
déduction du temps passé en & tutuklulukta geçen süre hapis \\
détention provisoire. » & cezasından indirilmiştir. » \\
(Affaire De Jong, Baljet et Van Den & (Traduction de Osman Doğru) \\
Brink contre Pays-Bas, 1984) & \\
(paragraphe 29) &
\end{tabular}

L'emploi de l'implicitation en traduisant en turc le terme de détention provisoire peut compromettre l'interprétation des textes traduits puisque la dimension temporaire n'est pas exprimée clairement; il est important de rendre 
Kurmel, D. (2017). Traduction en Turc de l'article 5 de la convention Europeenne

des droits de l'homme:differents yypes de la detention. Humanitas, 5(9), 29-43

explicitement en turc le caractère temporaire avec des modifieurs temporels comme les langues officielles, l'anglais et le français, le font.

\subsection{Détention préventive}

Détention préventive, un autre terme complexe, ayant un nombre d'occurrences significatif dans le corpus de jurisprudence en français n'est pas reflétée en turc, c'est-à-dire un terme complexe comme détention préventive ne figure pas dans le corpus de jurisprudence en turc, nous constatons que ce terme est rendu en turc par des variantes terminologiques de détention sans ajouter un modifieur qui insiste sur le caractère préventif. Dans la traduction en turc du terme complexe de détention préventive, nous constatons la stratégie de l'implicitation comme c'est le cas dans la traduction de la détention provisoire, c'est-à-dire le modifieur préventif est exprimé en turc de façon implicite.

Dans les extraits de l'affaire Wemhoff contre Allemagne (1968) tirés du corpus de jurisprudence (Tableau 2), nous constatons l'emploi du terme de détention préventive dans le contexte d'un risque de fuite et de collusion. En traduisant en turc cet extrait, le traducteur utilise simplement tutukluluk (détention) sans ajouter le modifieur préventif.

Tableau 2: Affaire Wemhoff contre Allemagne (1968) et sa traduction en turc

"Il convient d'ajouter qu'aux yeux de

la Commission, la détention préventive continue du requérant, ordonnée par les juridictions compétentes en raison du risque de fuite et de collusion, a été régulière au sens de l'article 5 par. 1 c) (art. 5 1-c).» (Affaire Wemhoff contre Allemagne, 1968) (section arguments de la Commission et du Gouvernement- paragraphe 12)

La détention provisoire et la détention préventive sont présentées comme des synonymes dans le Dictionnaire des Droits de l'Homme (2008) mais aussi en mettant en valeur des aspects complémentaires. Les équivalents en turc de ces termes complexes n'explicitent ni la dimension temporaire ni la dimension préventive.

\subsection{Détention sans jugement}

Dans les affaires de Lawless contre Irlande (1961) et d'Irlande contre RoyaumeUni (1978) de notre corpus, nous constatons un autre type de détention, qui a un statut différent par rapport à détention provisoire et à détention préventive considérées en tant que synonymes, et qui constitue la spécificité de ces affaires: détention sans jugement. Bien que détention sans jugement soit un terme qui est utilisé seulement dans deux arrêts, il nous paraît utile d'analyser ce terme complexe, emprunté aux droits internes d'Irlande et de Royaume-Uni 
Kurmel, D. (2017). Traduction en Turc de l'article 5 de la convention Europeenne des droits de l'homme:differents yypes de la detention. Humanitas, 5(9), 29-43

(detention without trail) afin de mettre en évidence comment ce concept qui n'est pas connu des autres systèmes juridiques est transféré dans les autres langues.

Ce type de détention qui s'exerce dans des conditions spéciales est expliquée comme ci-dessous dans l'affaire de Lawless contre Irlande (1961);

«Durant la période qui s'est écoulée depuis la création de l'État libre d'Irlande, des groupes armés se sont constitués à plusieurs reprises sous le nom d"Armée républicaine irlandaise" (I.R.A), dans le but avoué de se livrer à des activités terroristes afin de mettre un terme à la souveraineté que la Grande-Bretagne exerce sur l'Irlande du Nord. Les activités de ces groupes ont parfois pris des proportions telles que la législation ordinaire ne permettait plus d'en assurer une répression efficace. Le législateur a donc, à plusieurs reprises, conféré au Gouvernement des pouvoirs spéciaux permettant à celui-ci de faire face à la situation créée par ces activités illégales; au nombre de ces pouvoirs a figuré parfois le droit de détention sans jugement. »(Affaire Lawless contre Irlande, 1961) (paragraphe 6)

Dans le corpus de jurisprudence en français, ce terme complexe détention sans jugement a 9 occurrences dans les deux arrêts que nous avons déjà mentionnés. Dans le corpus de jurisprudence en turc, nous constatons que ce terme a été traduit par la combinaison du terme tutma (détention) et de l'adjectif yargisiz / yargilamasız (sans jugement) comme dans les langues officielles. Donc, nous constatons un équivalent assez complet pour le terme détention sans jugement.

Un des emplois de yargilamasız tutma dans le corpus figure accompagné du terme de la langue source anglais, detention without trial. Le traducteur ajoute une information supplémentaire en gardant le terme du texte officiel en anglais entre parenthèses dans la traduction de l'extrait du paragraphe 6 de l'affaire de Lawless contre Irlande (1961) où le terme se définit, car le terme de la langue officielle fait référence à un nouveau concept pour la langue cible qui est le turc, pour cette raison le traducteur mentionne aussi le terme original avec son équivalent en turc.

«Serbest Irlanda Devletinin kuruluşundan bu yana, Kuzey Irlanda'daki Britanya egemenliğine son verme amaçlarını şiddet eylemleriyle gerçekleştireceklerini açılkayan ve kendilerine "İrlanda Cumhuriyetçi Ordusu" (IRA) adinı veren silahl gruplar oluşmuştur. Bu grupların eylemleri bazen o hale gelmiştir ki, bunları olağan hukuk usulleriyle etkili bir biçimde bastırmak mümkün olamamıştır. Bu nedenle yasama organı, yasadışı eylemlerin yarattığl durumu ortadan kaldırabilmesi için Hükümete zaman zaman, yargllamasız tutma (detention without trial) yetkisini de içeren bazı özel yetkiler vermiştir.» (Affaire Lawless contre Irlande, 1961) (paragraphe 6) (Traduction de Osman Doğru)

Dans la traduction en turc du terme complexe, détention sans jugement, nous constatons une différence significative par rapport à la traduction des termes détention provisoire et détention préventive parce que l'implicitation n'est pas 
Kurmel, D. (2017). Traduction en Turc de l'article 5 de la convention Europeenne des droits de l'homme:differents yypes de la detention. Humanitas, 5(9), 29-43

adoptée comme une stratégie au sein des traductions du terme détention sans jugement. C'est sans doute normal dans un sens: la détention est censée être provisoire, le caractère provisoire est présent dans le contenu sémantique de la détention, donc on ne le précise pas dans les traductions en turc, mais la détention sans jugement est un cas particulier, «sans jugement » ne figure pas dans le contenu sémantique de la détention, il est donc normal que ce soit bien précisé.

\subsection{Détention administrative}

Détention administrative qui une de détention sans jugement que certains pays exercent afin d'assurer la sécurité dans le pays, est employée au sein de l'affaire de Lawless contre Irlande (1961). Dans le corpus de jurisprudence en turc, nous observons que ce terme est traduit en turc par deux équivalents idari gözaltında tutma (détention administrative) et idari tutma (détention administrative), formés à partir de deux variantes terminologiques de détention qui sont tutma et gözaltında tutma ainsi que l'adjectif idari (administratif). Ces deux termes complexes en turc sont utilisés de façon identique en tant qu'équivalent du terme de détention administrative (Tableau 3 ).

Comme les extraits ci-dessous tirés de nos corpus nous démontrent, le traducteur préfère accompagner ce terme de son équivalent en anglais administrative detention en vue de rendre plus compréhensible ce terme avec cette indication supplémentaire.

Tableau 3: Affaire Lawless contre Irlande (1961) et sa traduction en turc

\begin{tabular}{|c|c|}
\hline $\begin{array}{l}\text { "Qu'il résulte de ce qui précède } \\
\text { qu'aucun des moyens } \\
\text { susmentionnés n'aurait pu } \\
\text { permettre de faire face de } \\
\text { manière efficace à la situation } \\
\text { existante en Irlande en 1957; } \\
\text { que, dans ces conditions, la } \\
\text { détention administrative - telle } \\
\text { qu'introduite par la Loi no } 2 \text { de } \\
1940-\text { des individus } \\
\text { soupçonnés de vouloir } \\
\text { participer à des entreprises } \\
\text { terroristes, se présentait, } \\
\text { malgré sa gravité, comme une } \\
\text { mesure exigée par les } \\
\text { circonstances. " } \\
\text { (Affaire Lawless contre Irlande, }\end{array}$ & $\begin{array}{l}\text { «Buradan çıan sonuca göre yukarıda } \\
\text { belirtilen araçlardan hiç biri 1957'de, } \\
\text { Irlanda'da varolan durumla mücadeleyi } \\
\text { mümkün kılamayacaktı. Bu nedenle, terörist } \\
\text { faaliyetlerde bulunma niyetinden } \\
\text { kuşkulanılan kişileri, } 1940 \text { tarihli } \\
\text { (Değişiklik) Yasastyla getirilen idari } \\
\text { gö̈altında tutmanın (administrative } \\
\text { detention)ağırlı̆̆ına rağmen, koşulların } \\
\text { gerektirdiği bir önlem olarakgörünmüş̧ür.» } \\
\text { (Traduction de Osman Doğru) }\end{array}$ \\
\hline
\end{tabular}

Nous observons que détention administrative est traduit en turc en gardant la même structure que les langues officielles comme c'est le cas dans la traduction 
de la détention sans jugement, c'est-à-dire avec la combinaison de l'équivalent de la détention et du modifieur administrative. Bien que ces deux termes figurent comme citations dans le droit européen, nous constatons le manque de termes correspondants en turc.

\section{Conclusion}

Une méthodologie terminologique textuelle basée sur l'analyse contextuelle menée dans des corpus de jurisprudence peut apporter des éclaircissements au niveau des termes des droits de l'homme européen et de leurs transferts en turc puisque les termes trouvent leur sens et s'interprètent dans la jurisprudence de la Cour.

Dans ce travail, l'analyse contextuelle menée dans des corpus de jurisprudence nous donne l'occasion de récupérer de différents types de détention et leur transfert en turc. L'implicitation est une stratégie adoptée dans la traduction en turc des termes complexes tels que; détention provisoire et détention préventive; le sens des modifieurs en question est transféré en turc de façon implicite puisque les équivalents de la détention ont un caractère provisoire de façon implicite. Il est essentiel de souligner que ce n'est pas suffisant de disposer des termes dont le périmètre sémantique n'est pas précisé, surtout lorsqu'il s'agit de la durée d'une détention. Cette situation peut causer des interprétations abusives des textes traduits en turc. Par contre, les termes complexes comme détention sans jugement et détention administrative renvoient à des situations exceptionnelles ; ils sont traduits en turc de façon explicite avec l'utilisation des modifieurs yargisız/yargilamasız (sans jugement) et idari (administrative) puisque ces modifieurs donnent un nouveau statut à la détention.

Ce travail nous montre que la traduction en turc des types de détention négligent certaines distinctions que la langue officielle possède, en adoptant une stratégie d'implicite. Est-ce qu'il est possible de parler d'une relationnalité entre le transfert en turc de la détention et de son exécution ? Il sera très prétentieux de répondre à cette question en se basant seulement aux données linguistiques/terminologiques car au niveau de l'exécution de l'article 5, des différents acteurs prennent une place. Mais sûrement, le transfert en turc de l'article 5 ayant le même effet juridique que la langue source possède influe d'une façon positive la réception de cet article et donc son exécution. Il est possible de dire qu'un travail interdisciplinaire réunissant juristes, linguistes, terminologues et traducteurs peut proposer des méthodes afin de produire des textes ayant le même effet juridique que les langues sources possèdent. 
Kurmel, D. (2017). Traduction en Turc de l'article 5 de la convention Europeenne

des droits de l'homme:differents yypes de la detention. Humanitas, 5(9), 29-43

\section{Bibliographie}

Alouini, J. (2003). «Terminologie et lexicalisation dans le discours des droits de l'homme » in Mejri, S. (dir) L'Espace euro-méditerranéen: Une idiomaticité partagée, Actes du colloque international (Tome2). Tunisie: Hammamet 19, 20 \& 21 septembre 2003, 13-21.

Andriantsimbazovina J., Gaudin H., Marguenaud J-P., Rials S. et Sudre F. (dir.) (2008). Dictionnaire des droits de l'homme. Paris: Puf.

Baccouche, T. (2001). «L'autre, enjeu sémantique et culturel dans les concepts fondamentaux des droits de l'homme ». L'éloge de la différence: la voix de l'autre. Paris: Hachette Edicef, 49-53.

(2004). " Pour une méthodologie d'un dictionnaire bilingue (français-arabe) des droits de l'homme » in Mejri, S. et P. Thoiron (dir.) La terminologie, entre traduction et bilinguisme. Journée scientifique de formation et d'animation régionale, Tunisie: Hammamet le 14 octobre 2004, 8-13.

Bourigault, D. et Slodzian, M. (1999). «Pour une terminologie textuelle». Terminologies nouvelles 19, 29-32.

Delisle J., Lee-Jahnke H. et Cormier M. C. (dir.) (1999). Terminologie de la Traduction/ Translation Terminology/ Terminología de la Traduccion/ Terminologie der Übersetzung. Amsterdam/Philadelphia: John Benjamins.

Chodkiewicz C., Bourigault D. et Humbley J. (2002). «Making a workable glossary out of a specialised corpus. Term extraction and expert knowledge » in Altenberg, B. et Granger S. (ed) Lexis in Contrast Corpus based approaches. Amsterdam/Philadelphia: John Benjamins, 249-267.

Cortese, G. (2005). «Indeterminacy in «Rainbow» Legislation: the Convention on the Rights of the Child» in Bhatia, V.K. (dir.) Vagueness in Normative Texts. Bern, Peter Lang, 255-285.

Garre, M. (1999). Human Rights in Translation, Legal Concepts in Different Languages. Copenhague: Copenhagen Business School Press.

Gemalmaz, M.S. (2003). Insan Haklart Belgeleri I (Documents des droits de 1'Homme). İstanbul: Boğaziçi Üniversitesi Yayınevi.

Kurmel, D. (2014). Les droits de l'homme en turc: aménagement ou développement spontané? Thèse de doctorat en Sciences du Langage, Université Paris Diderot, (non publié).

Peshkov, K. (2012). Le discours juridique en russe et en français: une approche typologique. Thèse de doctorat en Langues, Lettres et Arts, Université d'Aix-Marseille. 
Kurmel, D. (2017). Traduction en Turc de l'article 5 de la convention Europeenne des droits de l'homme:differents yypes de la detention. Humanitas, 5(9), 29-43

Pic, E. (2007a). Caractérisation de l'anglais des droits de l'homme en tant que langage de spécialité. Un essai de méthodologie terminologique. Thèse de doctorat en Sciences du Langage, Université Paris Diderot.

(2007b). «Comprendre les concepts des droits de l'homme: ou terminologie et traductologie se rejoignent-elles? Une évaluation des théories cognitives. ». LA TILV, no 43, novembre 2007, 62-75.

(2008). « Normes culturelles et manières de traduire: le cas des droits de l'homme ». LA TILV, no 45, novembre 2008, 25-35.

Roets, D. (2008). « Détention provisoire» in Andriantsimbazovina J, Gaudin H., Marguénaud J-P., Rials S. et Sudre F. (dir.), Dictionnaire des droits de l'homme. Paris: Puf, 271-273.

Sudre, F. (2006). Droit européen et international des droits de l'homme. Paris: Puf, (8 $8^{\text {ème }}$ édition).

\section{Sites Internet}

http://www.antlab.sci.waseda.ac.jp/software.html: Concardancier AntConc [Accédé Janvier 2010]

http://hudoc.echr.coe.int: Base de données HUDOC

http://www.inhak.adalet.gov.tr: İnsan Hakları Daire Başkanlığı (Département des droits de l'homme)

http://www.inhak.adalet.gov.tr/mevzuat/mevzuat.html: Traductions de la jurisprudence diffusé dans le Département des droits de l'homme [Accédé Janvier 2012]

http://ihami.anadolu.edu.tr: Anadolu Üniversitesi Hukuk Fakültesi İnsan Hakları Hukuku Projesi, İnsan Hakları Avrupa Mahkemesi İçtihatları (Projet des droits de l'Homme - la jurisprudence de la Cour européenne des droits de l’Homme) [Accédé Janvier 2011]

www.resmigazete.gov.tr: Journal Officiel, 19/03/1954, no. 8662 et Journal Officiel, 20/06/1997, no. 23025 [Accédé Avril 2013]

\section{TRANSLATION OF ARTICLE 5 OF THE EUROPEAN CONVENTION ON HUMAN RIGHTS: DIFFERENT TYPES OF DETENTION (DÉTENTION)}

Abstract: Translation has an important place in the field of European human rights since the official languages of the European Convention on Human Rights and the jurisprudence of the European Court of Human Rights are English and French. The member states of the Convention transfer these texts into their languages via translation. In the translation of European human rights, the transposition of concepts with characteristics related to this field merits reflection; the new terminological approaches that take the terms in consideration within their discursive environment can offer a methodology in the translation of human rights, particularly in the transfer of the terms of the field which take their meaning in the texts of the jurisprudence. In this article, we 
Kurmel, D. (2017). Traduction en Turc de l'article 5 de la convention Europeenne des droits de l'homme:differents yypes de la detention. Humanitas, 5(9), 29-43

problematize the translation of the European human rights into Turkish through the translation of the different types of term detention (détention), which is the key term of the Article 5 of the European Convention on Human Rights "Right to liberty and security". We adopt a textual terminology approach based on the contextual analysis carried out in corpuses composed of French and Turkish jurisprudence texts in order to examine different types of the term detention (détention) such as détentionprovisoire, détentionpréventive, détention sans jugement, détention administrative, and their translations into Turkish.

Keywords: Translation of European Human Rights, Textual Terminology, Contextual Analysis, Corpus Analysis, Detention. 